\title{
Separate Inhibitory and Excitatory Components Underlying Receptive Field Organization in Superficial Medullary Dorsal Horn Neurons
}

\author{
Go Kato, Masafumi Kosugi, Masaharu Mizuno, and Andrew M. Strassman \\ Department of Anesthesia and Critical Care, Beth Israel Deaconess Medical Center and Harvard Medical School, Boston, Massachusetts 02215
}

Extracellular recording has shown that dorsal horn neurons can have an inhibitory surround outside their excitatory receptive field, but cannot reveal inhibitory inputs within the excitatory field, or show the underlying excitatory and inhibitory synaptic inputs that determine net output. To study the underlying components of receptive field organization, in vivo patch-clamp recording was used to compare the size and distribution of subthreshold, suprathreshold, and inhibitory fields, in neurons in the mouse superficial medullary dorsal horn that were characterized by their responses to noxious and innocuous mechanical facial stimulation. Subthreshold excitatory fields typically extended some distance beyond the borders of the suprathreshold field, and also commonly exhibited broader stimulus selectivity, in that the majority of nociceptive-specific neurons exhibited subthreshold responses to brush. Separate voltage-clamp recording of excitatory and inhibitory inputs using different holding potentials revealed that inhibition could be evoked from both within and outside the excitatory field. In nociceptive neurons, inhibition tended to be maximal at the excitatory receptive field center, and was usually greater for pinch than brush, although the selectivity for pinch versus brush was not as great as with excitatory responses. Based on current data on dorsal horn organization, we propose that the localized peak of inhibition at the excitatory field center could be mediated by local interneurons, while the more widespread surrounding inhibition may depend on supraspinal circuitry.

\section{Introduction}

Neurons of the spinal and medullary dorsal horn typically exhibit a receptive field on a region of the body surface from which they can be driven to fire by one or more forms of noxious or innocuous stimulation. In addition to such excitatory receptive fields, dorsal horn neurons can exhibit surrounding inhibitory fields from which firing can be suppressed by innocuous or noxious stimulation (Mendell and Wall, 1965; Hillman and Wall, 1969; Wagman and Price, 1969; Christensen and Perl, 1970; Besson et al., 1974; Le Bars et al., 1979). The receptive field properties of dorsal horn neurons may be viewed as representing the net balance between excitatory and inhibitory influences from the periphery, and the relative strength of each these two opposing influences may vary across different regions of the receptive field. The spatial organization of the resulting receptive field is thus a product of this spatial variation in the two underlying components. Extracellular unit recording, the primary method for the study of receptive field properties, shows the discharge or net output of the neuron, but not the separate underlying excitatory and inhibitory components of synaptic input that determine the net output.

\footnotetext{
Received Sept. 1, 2011; revised 0ct. 7, 2011; accepted 0ct. 12, 2011.

Author contributions: G.K. and A.M.S. designed research; G.K. and M.K. performed research; G.K., M.M., and A.M.S. analyzed data; G.K. and A.M.S. wrote the paper.

This work was supported by the National Headache Foundation and by NIH Grant R01 NS057454 to A.M.S.

Correspondence should be addressed to Andrew Strassman, Department of Anesthesia, CLS 647, Beth Israel Deaconess Medical Center, 3 Blackfan Circle, Boston, MA 02215. E-mail: Andrew_Strassman@bidmc.harvard.edu. DOI:10.1523/JNEUROSCI.4474-11.2011

Copyright $\odot 2011$ the authors $\quad 0270-6474 / 11 / 3117300-06 \$ 15.00 / 0$
}

In extracellular recordings, inhibitory fields are detected indirectly, as a suppression of discharge, making it difficult to quantify the variation in the strength of inhibitory effects across the receptive field. Inhibitory effects evoked from within the excitatory field can in some cases be detected as a suppression of discharge following the initial excitation, when relatively weak excitatory stimuli are used (Hillman and Wall, 1969), but are likely to become obscured by more powerful excitatory stimuli. Thus, from such studies, it is unclear to what degree the inhibitory pattern is actually a surround inhibition, arising preferentially from regions outside the excitatory receptive field, or instead whether there might be an equal or greater amount of inhibitory input arising from within the excitatory field. Intracellular recording of membrane potential allows a more direct observation of the excitatory and inhibitory synaptic inputs underlying receptive field organization, but still has the limitation that membrane potential changes represent a combination or summation of excitation and inhibition, and so it can be difficult to measure inhibitory inputs when they are evoked from within the excitatory receptive field. Patch-clamp recording, when done in voltage-clamp mode, has the advantage of allowing the measurement of excitatory or inhibitory inputs in isolation. In the present study, to study the underlying components of receptive field organization, in vivo patch-clamp recording was used to examine both subthreshold excitatory inputs and inhibitory inputs, and to compare the size and distribution of these fields with the suprathreshold receptive fields, in a sample of neurons recorded in the superficial part of the medullary dorsal horn in the mouse that were characterized by their 
responses to noxious and innocuous mechanical stimulation of the face.

\section{Materials and Methods}

Methods for in vivo patch-clamp recording were similar to those performed previously in the spinal dorsal horn by Kato et al. (2006). The experimental protocol was approved by the Institutional Animal Care and Use Committee of the Beth Israel Deaconess Medical Center. Adult male mice (C57BL/6, 4-6 weeks) were anesthetized with urethane (1.5 g/kg, i.p., Sigma). Adequacy of anesthetic depth was judged by suppression of corneal blink and hindlimb withdrawal reflexes. Core body temperature was maintained at $37^{\circ} \mathrm{C}$ by a feedback-controlled heating pad (FHC). The animal was placed in a stereotaxic apparatus and further stabilized with vertebral clamps (Narishige SR-5M and STS-A). Part of the $\mathrm{C} 1$ bone and the ventral portion of the occipital bone were removed to expose the caudal brainstem and uppermost portion of the cervical spinal cord. An incision was made in the dura. Skin flaps on either side of the exposure were pulled upward with hooks.

Patch micropipettes $(6-10 \mathrm{M} \Omega$ ) were made from borosilicate glass with a Sutter puller and filled with (in mM) $110 \mathrm{Cs}_{2} \mathrm{SO}_{4}, 5 \mathrm{TEA} \mathrm{Cl}, 0.5$ $\mathrm{CaCl}_{2}, 2 \mathrm{MgCl}_{2}, 5$ EGTA, 5 HEPES, $5 \mathrm{Mg}$ ATP, $0.1 \%$ Neurobiotin, and either (1) $136 \mathrm{~K}$ gluconate and $5 \mathrm{KCl}$ or (2) $110 \mathrm{Cs}_{2} \mathrm{SO}_{4}$.

Micropipettes were advanced with a motorized micromanipulator (MP-225, Sutter) through the superficial laminae of the medullary dorsal horn in a posterior-to-anterior direction, at a vertical angle of $45^{\circ}$ relative to the medullary surface. Positive pressure was used to prevent blockage during penetration of the surface. After penetration of the most dorsal white matter, to improve stability, a pool was filled with $3 \%$ agarose in Krebs' solution equilibrated with $95 \% \mathrm{O}_{2}-5 \% \mathrm{CO}_{2}$, containing the following (in mM): $117 \mathrm{NaCl}, 3.6 \mathrm{KCl}, 2.5 \mathrm{CaCl}_{2}, 1.2 \mathrm{MgCl}_{2}, 1.2 \mathrm{NaH}_{2} \mathrm{PO}_{4}$, 11 glucose, and $25 \mathrm{NaHCO}_{3}$. Spontaneous discharge was mostly absent. The presence of a neuron was detected by a change in series resistance. Pulses of depolarizing current were used to monitor series resistance and detect the formation of a gigaohm seal. After formation of a gigaohm seal, evoked spikes were monitored under cell-attached mode for cell classification (see below). Additional suction was then used to attain whole-cell recording configuration. All encountered neurons were studied. In voltage-clamp recordings, excitatory (inward) currents were recorded at a holding potential of $-70 \mathrm{mV}$. Inhibitory (outward) currents were recorded at a holding potential of $0 \mathrm{mV}$. Attainment of this holding potential usually requires cesium in the internal solution (see above) for improved voltage-clamp control. Membrane potentials were not corrected for the liquid junction potential between the Krebs and patch pipette solutions.

Neurons were tested for responses to noxious and innocuous mechanical stimulation (pinch and brush) of cutaneous facial receptive fields, as well as brush of cornea, which was considered a noxious stimulus. Stimulus duration was $\sim 5 \mathrm{~s}$. For receptive field mapping, stimuli were delivered at $30 \mathrm{~s}$ intervals. Neurons were classified based on their suprathreshold responses to cutaneous stimulation as nociceptive specific (NS, discharge to pinch only), wide dynamic range (WDR, discharge greater to pinch than brush), or low threshold (maximal discharge in response to brush). Discharge was quantified as the mean rate during the stimulus. Some neurons had a substantial pinch discharge that was as large as or slightly larger than their brush discharge, but were nonetheless classified as low threshold because the pinch discharge was transient $(<0.5 \mathrm{~s}$ duration $)$, which is not considered characteristic of nociceptive neurons. EPSC and IPSC responses were quantified as the mean current recorded during the stimulus, minus the mean current recorded for an equal duration immediately preceding the stimulus.

Signals were amplified and filtered at $4 \mathrm{kHz}$ (Multiclamp 700B, Molecular Devices) and sampled at $10 \mathrm{kHz}$, digitized, and acquired with Digidata 1440A and Clampex 10.1 (Molecular Devices). Signals were also played through a speaker to aid in receptive field mapping. Data were analyzed with the aid of Clampfit 10.1 (Molecular Devices).

Neurons were labeled by diffusion of Neurobiotin from the patch pipette. Animals were perfused with $4 \%$ paraformaldehyde in phosphate buffer, and after postfixation, tissue blocks were cut into transverse sec- tions of $60-70 \mu \mathrm{m}$ on a freezing microtome, and stained for Neurobiotin using Alexa 488-conjugated streptavidin.

\section{Results}

Recordings were obtained from neurons in the superficial laminae of the medullary dorsal horn of anesthetized mice using patch micropipettes. Receptive field responses were studied using two different recording paradigms, in separate neuronal samples: (1) whole-cell recording of membrane potential $(n=26)$; and (2) recording of spikes in cell-attached mode under current clamp, followed by recording of EPSCs, or IPSCs, or both, in whole-cell mode under voltage clamp (i.e., spikes, EPSCs, and IPSCs, each recorded in a separate trial $)(n=15)$. In a subset of the first group, and all neurons of the second group, the receptive field was mapped by stimulating at sites in a standardized stimulation grid (see Fig. 2A).

Neurons were tested for responses to noxious and innocuous mechanical stimulation (pinch and brush) of cutaneous facial receptive fields, as well as brush of cornea, which was considered a noxious stimulus. Neurons were classified based on their suprathreshold responses to cutaneous stimulation as nociceptive specific (NS; $n=13$ ), wide dynamic range (WDR; $n=$ 17), or low threshold (LT; $n=11$ ). A total of 9 neurons had a suprathreshold cornea response, and 7 others had a subthreshold cornea response. Soma locations (see Fig. 2C) were recovered for 23 Neurobiotin-stained neurons (lamina I, $n=9$; lamina II, $n=11$, II-III border region, $n=3$ ).

\section{Subthreshold excitatory responses}

Subthreshold responses could commonly be evoked from stimulus sites that were outside the suprathreshold receptive field; this was found for both brush and pinch. In addition, subthreshold fields also differed from suprathreshold fields in their stimulus selectivity, in that the majority of nociceptive-specific neurons (9/13) exhibited subthreshold responses to brush. In whole-cell recordings of membrane potential (recording paradigm no. 1 above), such subthreshold responses appeared as subthreshold depolarizations (Fig. 1 $A a$ ). In voltage-clamp recordings, subthreshold responses were identified as an increased inward current (EPSCs) evoked from a stimulus site from which no spikes had been evoked during the cell-attached recording trial (recording paradigm no. 2 above; e.g., compare spike and EPSC maps in Fig. 2A3).

\section{Inhibitory responses}

In addition to suprathreshold and subthreshold depolarizations, hyperpolarizations (presumed IPSPs) were evoked by facial stimulation in most neurons during whole-cell recordings of membrane potential. Hyperpolarizations could be evoked not only from stimulus sites outside, but also from within the excitatory receptive field, in which case they appeared as an afterhyperpolarization that followed the EPSPs or action potentials (Fig. $1 B$ ). However, the presence of depolarizations interferes with both the detection and the measurement of the hyperpolarizations evoked from within the excitatory field, making it difficult to compare the amplitude of hyperpolarizations to those outside the excitatory field, and thus determine the overall distribution of the inhibitory input field. Voltage-clamp recording has the advantage that it can show excitatory and inhibitory inputs separately by the use of appropriate holding potentials, thus making it possible to examine the degree of inhibition occurring not only in the surround, but also within the excitatory receptive field, without having the inhibition obscured by concurrent excitation. 

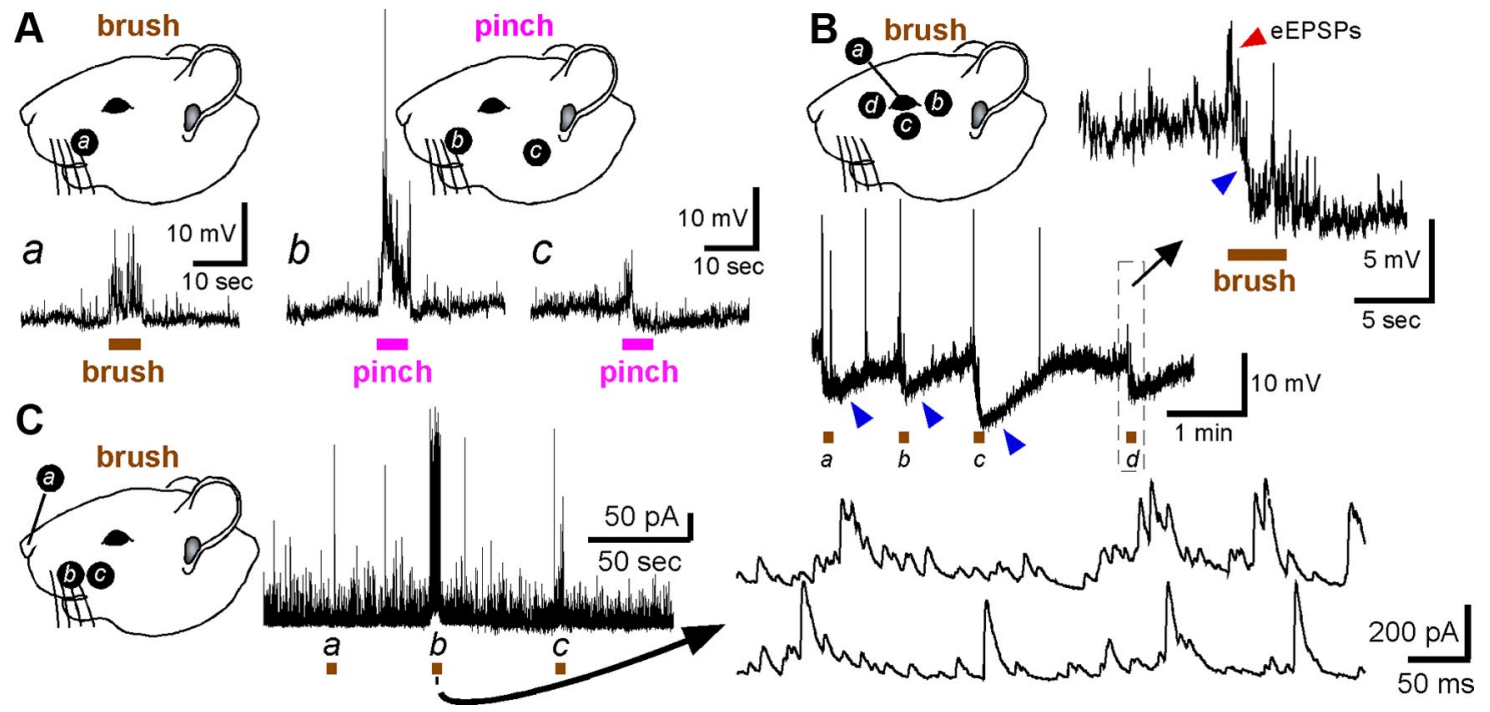

Figure 1. Examples of whole-cell recordings of responses of superficial medullary dorsal horn neurons to facial brush and pinch under current clamp ( $\boldsymbol{A}$, lamina II NS neuron; $\boldsymbol{B}$, lamina I WDR neuron) or voltage clamp (C, IPSC recording, $0 \mathrm{mV}$ holding potential). Blue arrowheads in $\boldsymbol{B}$ indicate afterhyperpolarizations following action potentials or EPSPs. Brown bars indicate brush.
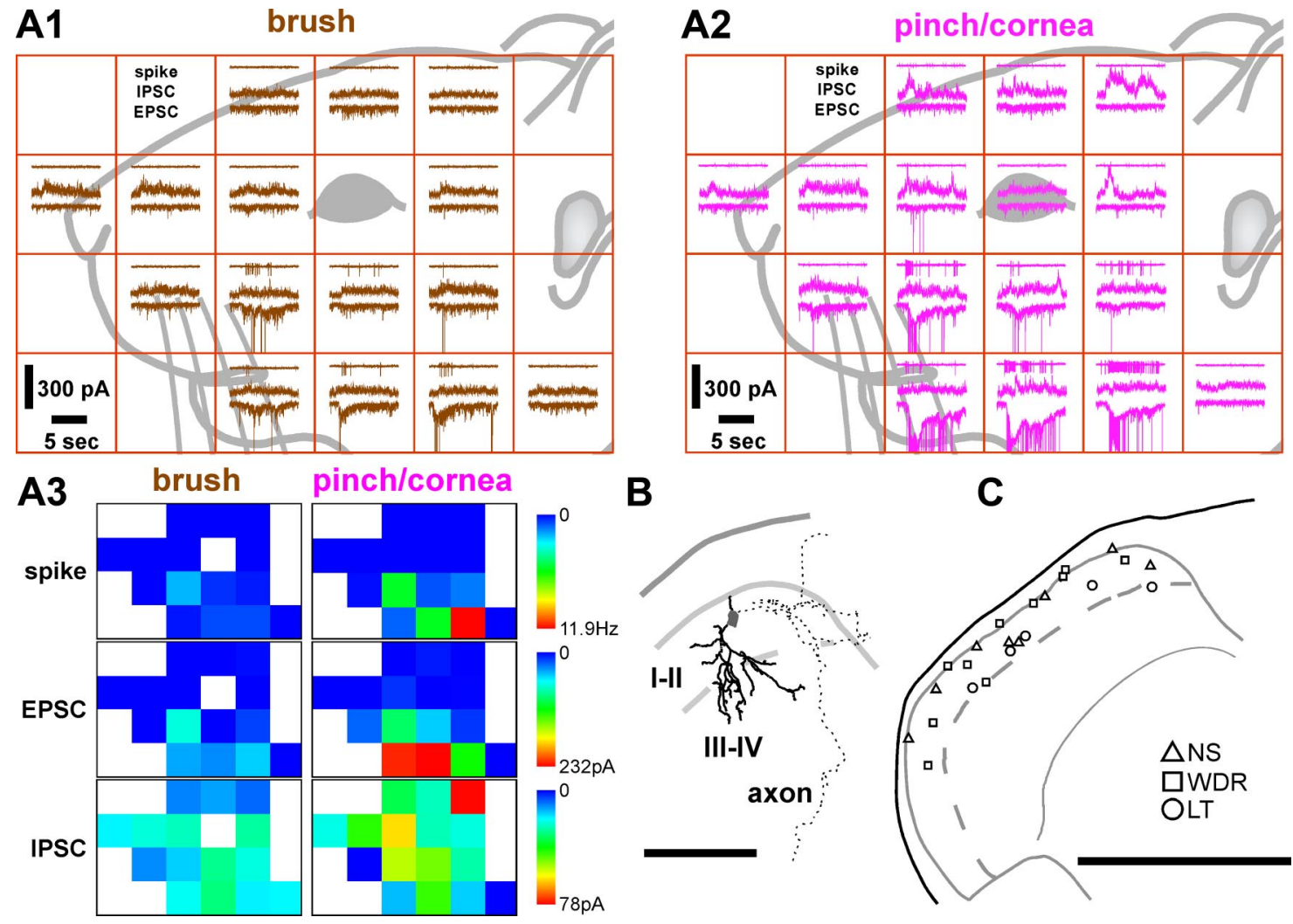

Figure 2. A1-A3, Method of receptive field mapping by delivering brush and pinch stimuli in a standardized grid of 16 facial sites, illustrated in a lamina II WDR neuron. Mapping was performed under three different recording conditions, in the following order: (1) recording of spikes in cell-attached mode, (2) whole-cell voltage-clamp recording of EPSCs at $-70 \mathrm{mV}$ holding potential, and (3) whole-cell voltage-clamp recording of IPSCs at $0 \mathrm{mV}$ holding potential. In each recording condition, mapping was first completed for brush at all sites, and then for pinch. Cornea was stimulated only with brush. Traces of the responses from each of the three recording conditions (spike, IPSC, and EPSC displayed from top to bottom on each facial site) are overlaid on the sites in the stimulus grid during brush (A1) and pinch (A2). $\mathbf{A}$, Color-coded maps showing the response amplitude at each site in the stimulation grids shown in $\boldsymbol{A} \mathbf{1}$ and $\boldsymbol{A} \mathbf{2}$, for each of the three recording conditions. Since corneal brush is considered a noxious stimulus, the cornea response is shown on the pinch maps, and the corneal site is blank on the brush maps. $\boldsymbol{B}$, Transverse somatodendritic and axonal reconstruction in transverse plane of the same neuron. Lateral is to the left. Scale bar, $200 \mu \mathrm{m}$. C, Locations of recorded Neurobiotin-stained neurons. Scale bar, $500 \mu \mathrm{m}$.

Figure 2 illustrates an example of a neuron that was studied using cell-attached recording of spikes followed by whole-cell voltage clamp, and stimulated with the standardized stimulation grid to facilitate comparison between the spatial extent of the suprathreshold, EPSC, and IPSC fields. Figure $3 A$ illustrates av- eraged maps of the entire sample of nociceptive neurons (nociceptive specific and wide dynamic range), in which the maps of individual neurons were aligned by their receptive field center. These maps show that the IPSC field tends to be broader than the EPSC field, for both brush and pinch. This difference in the 

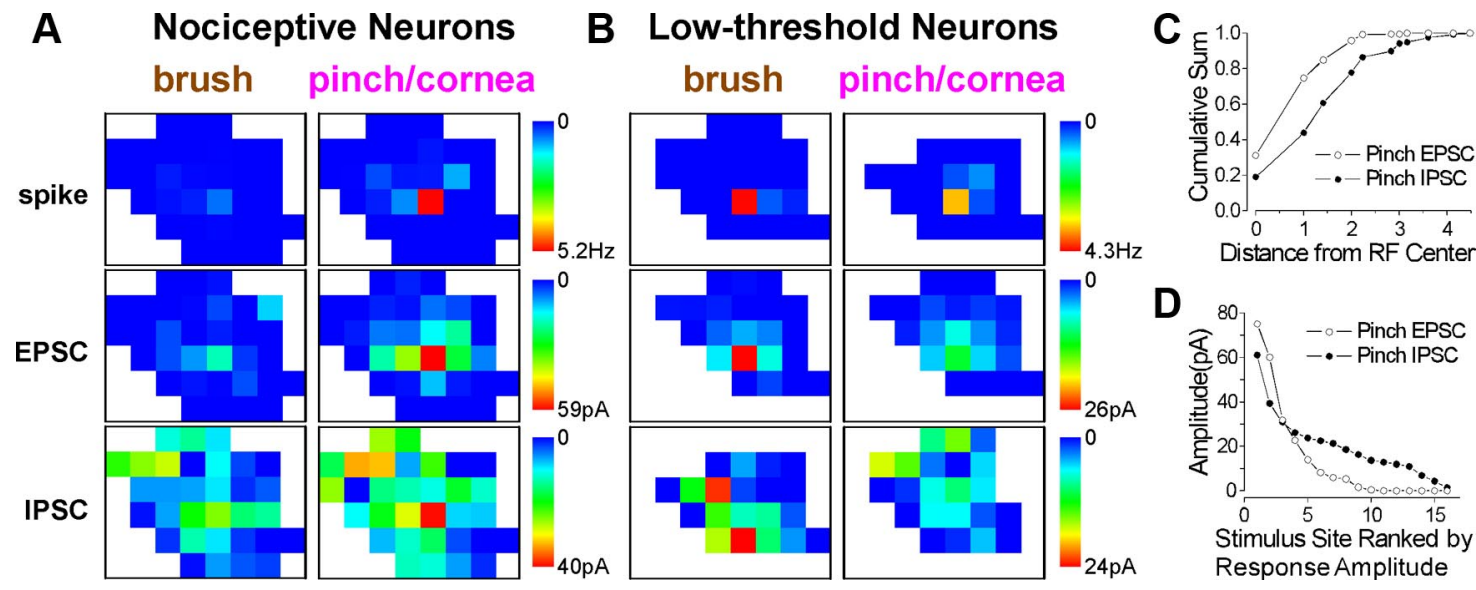

Figure 3. $\quad \boldsymbol{A}, \boldsymbol{B}$, Averaged receptive field maps showing response amplitude measured in separate recording trials for spikes, EPSCS, and IPSC, for the sample of nociceptive ( $\boldsymbol{A})$ and nonnociceptive $(\boldsymbol{B})$ neurons that were recorded in cell-attached mode followed by whole-cell voltage clamp (as illustrated in Fig. 2$)(\boldsymbol{A}, n=10 ; \boldsymbol{B}, n=5 ;$ but not all neurons were recorded for all 6 recording conditions). For averaging, the maps of individual neurons were shifted to align them by their receptive field "center" (site of maximum spikes). Therefore, sites in averaged maps do not correspond to the original facial sites, but to a position relative to the receptive field center, whose location on the face varies between neurons. This shifting of the individual maps is the reason that the number of sites in the averaged maps is larger than the number of sites in the individual maps (Fig. 2 A1). The sample of nociceptive neurons was too small to allow making separate averaged maps for subpopulations based on location (lamina I vs lamina II) or response type (NS vs WDR). C, D, Two types of plots showing that IPSC fields are broader than EPSC fields, for pinch stimuli in nociceptive neurons. C, Cumulative sum of all responses at all stimulus sites as a function of increasing distance from receptive field center (center defined as site of maximum spike response), in the subset of neurons that were stimulated at multiple sites in a standardized grid. Distance unit is the distance between adjacent sites on the grid map (Fig. $2 A 1-A 2$ ). Curves show distribution is broader for IPSCs than EPSCS ( $p<0.05$, repeated-measures ANOVA). D, Plot of mean response amplitude for each site in the stimulus grid, ranked in order of stimulus amplitude.

broadness of the fields is illustrated graphically in the plots in Figure $3 C$. The averaged maps of the IPSC field also shows that, although the averaged IPSC field is more broadly distributed than the EPSC field, it nonetheless has its maximum at the center (maximum site) of the excitatory field. The IPSC field thus has substantial components both within and outside the excitatory field. The averaged maps for low-threshold neurons similarly show a broader IPSC field than EPSC field (Fig. $3 B$ ); in contrast to the nociceptive neurons, the low-threshold neurons showed greater inhibition from brush than pinch.

In the membrane potential data described above, inhibition was more readily seen with brush than with pinch, possibly because the large excitatory responses to pinch in nociceptive neurons tend to obscure hyperpolarizing responses; however, with voltage-clamp recordings, inhibition could be readily seen with pinch as well as brush, both within and outside the excitatory receptive field. The averaged maps show that, in nociceptive neurons, inhibition from pinch is greater than from brush, although the selectivity for pinch over brush is not as great for IPSCs as for EPSCs or spike responses; some nociceptive neurons had somewhat greater inhibition from brush than pinch. No correlation was found between the amplitude of the EPSC response and the amplitude of the IPSC response.

\section{Discussion}

The present study used in vivo patch-clamp recording to examine the separate excitatory and inhibitory synaptic components underlying receptive field spatial organization for a sample of neurons in the superficial laminae of the medullary dorsal horn. To facilitate comparison of the spatial distribution of suprathreshold, excitatory, and inhibitory input fields, a standardized stimulation grid was used for receptive field mapping, using innocuous and noxious (brush and pinch) facial stimuli. Similar to findings in deep dorsal horn neurons (Woolf and King, 1989), the present sample of superficial neurons exhibited subthreshold excitatory fields that typically extended some distance beyond the borders of the suprathreshold field, and also commonly exhibited broader stimulus selectivity, in that the majority of nociceptivespecific neurons exhibited subthreshold responses to brush. Separate voltage-clamp recordings of excitatory and inhibitory inputs using different holding potentials revealed that inhibitory synaptic inputs could be evoked from both within and outside the excitatory receptive field, and, for nociceptive neurons (neurons that fired either preferentially or exclusively to noxious stimuli), the inhibition was maximal at the center of the excitatory receptive field, when viewed in receptive field maps obtained by averaging across neurons. Nociceptive neurons (but not non-nociceptive neurons) tended to show greater inhibition from noxious than innocuous stimuli, but the difference was much less than for excitatory inputs. Narikawa et al. (2000), in a sample of lamina II neurons in the lumbar dorsal horn, found that the majority of neurons had strong IPSC responses to brush but not pinch, but that study did not classify the neuronal sample by firing responses. There appears to be no prior study in which recording of firing properties was combined with recording of synaptic currents (either EPSC or IPSC) in a sample of dorsal horn neurons.

\section{Subthreshold responses}

Central sensitization is a state of increased responsiveness that can be induced in some types of nociceptive dorsal horn neurons by sustained noxious input. It can be expressed not only as an augmentation of responses, but also as the appearance of novel responses to inputs from previously ineffective stimulus modalities (i.e., brush) or previously ineffective stimulus sites. It has been shown for deep dorsal horn neurons that such inputs can be already present at a subliminal level in the baseline state (Woolf and King, 1989), and so the suprathreshold expression of these inputs in the sensitized state only requires the enhancement of already existing inputs, or an increase in excitability (Woolf and King, 1990). The present results show that such subliminal inputs, particularly innocuous inputs in nociceptive-specific neurons, are also commonly present in neurons of superficial laminae. Subliminal inputs, which 
could be enhanced by depolarizing current, were also observed by Weng and Dougherty (2002). Enhancement of such responses might be one mechanism that contributes to the novel appearance of low-threshold responses that can be induced in nociceptive neurons of superficial laminae under conditions of central sensitization and pain hypersensitivity (Woolf et al., 1994; Keller et al., 2007).

\section{Inhibitory responses}

Previous studies of nociceptive neurons in both superficial and deep dorsal horn have found inhibitory effects from activation of fast-conducting low-threshold mechanoreceptive primary afferents, as initially proposed in the gate control theory (Melzack and Wall, 1965), as well as additional inhibitory effects, which are typically more powerful and widespread, from activation of slower conducting $(\mathrm{A}-\delta$ and $\mathrm{C}$ ) nociceptive primary afferents (Mendell and Wall, 1965; Hillman and Wall, 1969; Wagman and Price, 1969; Christensen and Perl, 1970; Besson et al., 1974; Handwerker et al., 1975; Cervero et al., 1979; Le Bars et al., 1979; Woolf, 1983; Narikawa et al., 2000; Kato et al., 2004). Hillman and Wall (1969) demonstrated that electrical stimulation within the excitatory receptive field of multireceptive deep dorsal horn neurons could evoke a mixture of both excitation and inhibition, where the inhibition manifested as a suppression of background discharge following the initial excitation. This inhibition was mostly apparent with innocuous and not noxious stimulus intensities, but this may have been because the inhibitory phase tended to become obscured by the larger and more prolonged excitatory responses that were evoked by higher stimulus intensities. The recording methods used in the present study showed that both innocuous and noxious mechanical stimuli can evoke inhibition from sites within as well as outside the excitatory receptive field. They also demonstrate a postsynaptic mechanism for brush- and pinch-induced inhibition in superficial dorsal horn neurons (whereas the suppression of discharge observed in extracellular recordings could also result from presynaptic inhibition or from disfacilitation, meaning inhibition of an excitatory interneuron, which might not be located in the superficial dorsal horn). The results raise a question about which fiber class might mediate the brush-induced inhibition, since there appears to be as yet no evidence for A- $\beta$-mediated inhibitory synaptic inputs to superficial dorsal horn neurons, in slice experiments (Yoshimura and Nishi, 1995). The brush-induced postsynaptic inhibition might instead be mediated by low-threshold mechanoreceptive $\mathrm{A}-\delta$ or $\mathrm{C}$ fibers, or they might be mediated by A- $\beta$ fibers but dependent on a supraspinal loop, which would not be present in the slice.

One finding that could not be anticipated from previous studies was that the averaged inhibitory map shows a localized peak of inhibition evoked from the center of the excitatory field. This spatial pattern, consisting of a distinct central spot superimposed on a much broader, fairly flat distribution, suggests the possibility that the two components of the field are generated by different mechanisms: the central spot is generated by local ("segmental") circuitry within the dorsal horn, while the broader surround is generated by, or dependent on, supraspinal loops that activate diffusely organized descending inhibitory systems from the brainstem. Two previous observations suggest this possibility. First, prior extracellular recording studies of dorsal horn neurons have generally found that blockade of descending impulses attenuated inhibition evoked from both within and outside the excitatory receptive field (but as noted above, such studies could not investigate inhibition evoked by intense stimuli within the excitatory field) (Hillman and Wall, 1969). Second, our studies with the method of laser scanning photostimulation, which demonstrates the location of local interneurons that are presynaptic to a given dorsal horn neuron, found that superficial dorsal horn neurons receive local inhibitory synaptic input from a more restricted area of the dorsal horn than the zone from which they receive local excitatory input (Kato et al., 2009). This was a seemingly paradoxical result, since the superficial dorsal horn has a very precise somatotopic organization, and so it might be expected that the larger size of peripheral inhibitory receptive fields would be paralleled by a larger local inhibitory synaptic input zone. The present results provide a possible explanation for this paradox: the local circuitry in itself generates only a very localized inhibitory field, whereas the far more widespread surrounding component of the inhibitory field is generated by diffusely organized descending brainstem projections. This descending inhibition presumably could not be exerted through excitation of a local inhibitory interneuron, because such a local interneuron would itself have to have a widespread excitatory field, and such fields are not found in superficial dorsal horn neurons. Instead, the brainstem neuron would have to exert a direct inhibitory action (Kato et al., 2006), or act through presynaptic facilitation of transmitter release from a local inhibitory interneuron (Baba et al., 2000, Abe et al., 2009).

\section{References}

Abe K, Kato G, Katafuchi T, Tamae A, Furue H, Yoshimura M (2009) Responses to 5-HT in morphologically identified neurons in the rat substantia gelatinosa in vitro. Neuroscience 159:316-324.

Baba H, Shimoji K, Yoshimura M (2000) Norepinephrine facilitates inhibitory transmission in substantia gelatinosa of adult rat spinal cord (part 1 ): effects on axon terminals of GABAergic and glycinergic neurons. Anesthesiology 92:473-484.

Besson JM, Catchlove RFH, Feltz P, Le Bars D (1974) Further evidence for postsynaptic inhibitions on lamina $\mathrm{V}$ dorsal horn interneurons. Brain Res 66:531-536.

Cervero F, Iggo A, Molony V (1979) An electrophysiological study of neurons in the substantia gelatinosa Rolandi of the cat's spinal cord. Q J Exp Physiol Cogn Med Sci 64:297-314.

Christensen BN, Perl ER (1970) Spinal neurons specifically excited by noxious or thermal stimuli: marginal zone of the dorsal horn. J Neurophysiol 33:293-307.

Handwerker HO, Iggo A, Zimmermann M (1975) Segmental and supraspinal actions on dorsal horn neurons responding to noxious and nonnoxious skin stimuli. Pain 1:147-165.

Hillman P, Wall PD (1969) Inhibitory and excitatory factors influencing the receptive fields of lamina 5 spinal cord cells. Exp Brain Res 9:284-306.

Kato G, Furue H, Katafuchi T, Yasaka T, Iwamoto Y, Yoshimura M (2004) Electrophysiological mapping of the nociceptive inputs to the substantia gelatinosa in rat horizontal spinal cord slices. J Physiol 560:303-315.

Kato G, Yasaka T, Katafuchi T, Furue H, Mizuno M, Iwamoto Y, Yoshimura M (2006) Direct GABAergic and glycinergic inhibition of the substantia gelatinosa from the rostral ventromedial medulla revealed by in vivo patch-clamp analysis in rats. J Neurosci 26:1787-1794.

Kato G, Kawasaki Y, Koga K, Uta D, Kosugi M, Yasaka T, Yoshimura M, Ji RR, Strassman AM (2009) Organization of intralaminar and translaminar neuronal connectivity in the superficial spinal dorsal horn. J Neurosci 29:5088-5099.

Keller AF, Beggs S, Salter MW, De Koninck Y (2007) Transformation of the output of spinal lamina I neurons after nerve injury and microglia stimulation underlying neuropathic pain. Mol Pain 3:27.

Le Bars D, Dickenson AH, Besson JM (1979) Diffuse noxious inhibitory controls (DNIC). I. Effects on dorsal horn convergent neurones in the rat. Pain 6:283-304.

Melzack R, Wall PD (1965) Pain mechanisms: a new theory. Science 150:971-979.

Mendell LM, Wall PD (1965) Responses of single dorsal cord cells to peripheral cutaneous unmyelinated fibres. Nature 206:97-99. 
Narikawa K, Furue H, Kumamoto E, Yoshimura M (2000) In vivo patchclamp analysis of IPSCs evoked in rat substantia gelatinosa neurons by cutaneous mechanical stimulation. J Neurophysiol 84:2171-2174.

Wagman IH, Price DD (1969) Responses of dorsal horn cells of M. mulatta to cutaneous and sural nerve A and C fiber stimuli. J Neurophysiol 32:803-817.

Weng HR, Dougherty PM (2002) Tuning of membrane properties regulates subliminal synapses in dorsal horn neurons of intact rats. Exp Neurol 175:209-215.

Woolf CJ (1983) C-primary afferent fibre mediated inhibitions in the dorsal horn of the decerebrate-spinal rat. Exp Brain Res 51:283-290.

Woolf CJ, King AE (1989) Subthreshold components of the cutaneous mechanoreceptive fields of dorsal horn neurons in the rat lumbar spinal cord. J Neurophysiol 62:907-916.

Woolf CJ, King AE (1990) Dynamic alterations in the cutaneous mechanoreceptive fields of dorsal horn neurons in the rat spinal cord. J Neurosci 10:2717-2726.

Woolf CJ, Shortland P, Sivilotti LG (1994) Sensitization of high mechanothreshold superficial dorsal horn and flexor motor neurones following chemosensitive primary afferent activation. Pain 58:141155.

Yoshimura M, Nishi S (1995) Primary afferent-evoked glycine- and GABAmediated IPSPs in substantia gelatinosa neurones in the rat spinal cord in vitro. J Physiol 482:29-38. 